\title{
QUEEN'S
UNIVERSITY
BELFAST
}

\section{Experimental determination of mode I fracture parameters in orthotropic materials by means of Digital Image Correlation}

Cappello, R., Pitarresi, G., Xavier, J., \& Catalanotti, G. (2020). Experimental determination of mode I fracture parameters in orthotropic materials by means of Digital Image Correlation. Theoretical and Applied Fracture Mechanics, 108, [102663]. https://doi.org/10.1016/j.tafmec.2020.102663

Published in:

Theoretical and Applied Fracture Mechanics

Document Version:

Peer reviewed version

Queen's University Belfast - Research Portal:

Link to publication record in Queen's University Belfast Research Portal

Publisher rights

Copyright 2020 Elsevier

This manuscript is distributed under a Creative Commons Attribution-NonCommercial-NoDerivs License

(https://creativecommons.org/licenses/by-nc-nd/4.0/), which permits distribution and reproduction for non-commercial purposes, provided the author and source are cited.

\section{General rights}

Copyright for the publications made accessible via the Queen's University Belfast Research Portal is retained by the author(s) and / or other copyright owners and it is a condition of accessing these publications that users recognise and abide by the legal requirements associated with these rights.

Take down policy

The Research Portal is Queen's institutional repository that provides access to Queen's research output. Every effort has been made to ensure that content in the Research Portal does not infringe any person's rights, or applicable UK laws. If you discover content in the Research Portal that you believe breaches copyright or violates any law, please contact openaccess@qub.ac.uk. 


\title{
Experimental determination of mode I fracture parameters in
}

\section{orthotropic materials by means of Digital Image Correlation}

\author{
Riccardo Cappello ${ }^{1}$, Giuseppe Pitarresi ${ }^{1}$, José Xavier ${ }^{2}$, Giuseppe Catalanotti ${ }^{3, *}$ \\ ${ }^{1}$ Department of Engineering - Università degli Studi di Palermo, Viale delle Scienze Ed. 8, 90128 Palermo, Italy \\ ${ }^{2}$ UNIDEMI, Department of Mechanical and Industrial Engineering, NOVA School of Science and Technology, \\ Universidade NOVA de Lisboa, 2829-516 Caparica, Portugal \\ ${ }^{3}$ Advanced Composites Research Group (ACRG), School of Mechanical and Aerospace Engineering, Queen's \\ University Belfast, Belfast BT9 $5 A H, U K$
}

\begin{abstract}
The mode I fracture parameters for an orthotropic body are directly calculated from full-field deformation measurements provided by Digital Image Correlation (DIC). Three complementary and direct approaches are evaluated and compared: i) the determination of the Stress Intensity Factor (SIF) by fitting the displacement field using the analytical expression proposed by Lekhnitskii; ii) the determination of the J-Integral by using the Energy Domain Integral (EDI) formulation on the raw DIC data; and iii) the calculation of the J-Integral using the EDI approach on the displacement data fitted using Lekhnitskii's formulation. A comparative experimental study is performed by testing an IM7/8552 cross-ply laminate, and the effect of different parameters is analysed and discussed. The outcomes of this work show that, if an accurate choice of the parameters is performed, the different approaches lead to the same results.
\end{abstract}

Keywords: Digital Image Correlation, Stress Intensity Factor, J-Integral, Least Squares Fitting, Energy Domain Integral

${ }^{*}$ Corresponding author: g.catalanotti@qub.ac.uk 


\section{Introduction}

Advanced composites are orthotropic quasi-brittle materials whose mechanical behaviour can be modelled using Linear Elastic Fracture Mechanics (LEFM). The determination of fracture parameters, such as the Stress Intensity Factor (SIF), the Energy Release Rate (ERR), or the JIntegral, provides information on the stress field in the neighbouring of the crack tip. An accurate determination of the aforementioned parameters is necessary to assess the structural integrity of these materials and their structures. A direct determination of fracture parameters can be done using the information provided by Digital Image Correlation (DIC) [1], a full-field non-contact optical experimental technique which can provide displacement and strain fields with suitable spatial resolution and accuracy around the crack tip. It should be noted that the assumption of validity of LEFM for describing the quasi-brittle behaviour of most polymer composites leads to the equivalence of the fracture parameters mentioned above.

Subramanyam et al. [2], Yates et al. [3] and Harilal et al. [4] employed the Least Squares Fitting (LSF) method on DIC data to determine the SIF in isotropic materials. Their studies proved the accuracy and effectiveness of the LSF method. Moreover, they demonstrated that 2D DIC provided results comparable to the ones obtained using more sophisticated methods, as 3D DIC [2] or FEM-based calibration [1,2], provided that a judicious choice of the fitting and the DIC parameters is made, and the location of the crack tip is calculated with sufficient approximation $[2,5,6]$. Other authors [7] showed that the DIC technique, combined with high-speed digital cameras, is able to study the crack growth behaviour of a polymeric beam subjected to impact loading, managing to extract the SIF for both mode I and II using a LSF procedure.

Rabbiolini et al. [8] used the LSF approach in an anisotropic material (Haynes 230 superalloy) in order to calculate the mixed mode (I+II) SIF, obtaining a good agreement between experimental and fitted displacements, proving the validity of the proposed approach also for orthotropic materials. In addition, Mogadpalli et al. [9] evaluated the SIF in a unidirectional GFRP specimen, showing a 
strong dependence of the SIF on the number of terms used in the fitting procedure. Lee et al. [10] used 2D DIC and the LSF of the Lekhnitskii formulation to study the fracture behaviour of unidirectional composites, performing both quasi-static and dynamic tests.

DIC data can also be used to compute the J-Integral [11] expressed either as a contour or an area integral (the so-called Energy Domain Integral approach). Long before the diffusion of the DIC, authors such as Dadkhah et al. [12], starting from the crack tip displacement fields determined by Moire interferometry, evaluated the J-Integral, studying its path independency and the existence of a HRR field. Subramanyam et al. [2] compared both the area and contour approaches for the evaluation of the J-Integral and concluded that the use of the Energy Domain Integral approach provides more accurate predictions. Similar conclusions were obtained by other authors [8-11].

Montenegro et al. [17] calculated the J-Integral in orthotropic materials using the contour integral approach, and reported that a convergence on the predictions is obtained only if the integration path is taken far enough from the crack tip location. The authors also pointed out that one of the biggest advantages in using J-Integral is that knowing the exact crack tip location is not a strict requirement.

Catalanotti et al. [18] calculated the critical value of the J-Integral using the contour integral approach in order to determine the resistance curves (R-Curves) of IM7/8552 cross-ply laminates, and concluded that the procedure provided results that were in good agreement with the ones obtained using a different approach based on the Finite Element Method (FEM).

Although several approaches have been attempted in determining the aforementioned fracture parameters using the DIC, a study on the different approaches (Least Squares Fitting and Energy Domain Integral methods) is missing in terms of systematic comparison and efficiency.

Hence, the aim of this work is to analyse these different approaches, in order to provide useful recommendations for the improvement of the accuracy and reliability of the methodology based on full-field deformation measurements provided by digital image correlation. 


\section{Theory}

\subsection{Evaluation of the SIF by fitting the displacement field}

The SIF of a two-dimensional orthotropic body containing a crack (Figure 1) can be determined by using the analytical expression for the displacements obtained by Lekhnitskii [19].

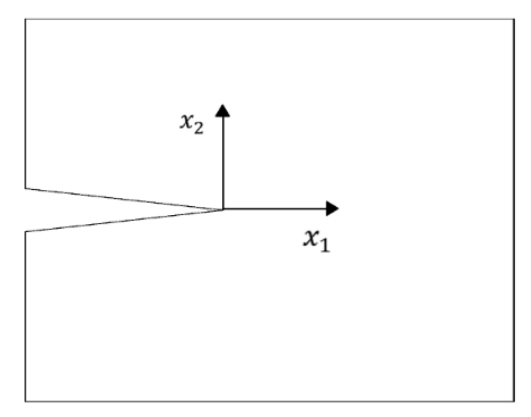

Figure 1-Cracked body with reference system centred on the crack-tip.

The first step consists in evaluating the complex material properties, $\mu_{i}$, as the roots of the characteristic equation:

$$
a_{11} \mu^{4}-2 a_{16} \mu^{3}+\left(2 a_{12}+a_{66}\right) \mu^{2}-2 a_{26} \mu+a_{22}=0
$$

where $a_{i j}$ are the terms of the compliance matrix in the Hooke's law, which, for plane stress, can be expressed in term of the engineering elastic constants:

$$
a_{11}=\frac{1}{E_{1}} ; a_{22}=\frac{1}{E_{2}} ; a_{12}=-\frac{v_{12}}{E_{1}}=-\frac{v_{21}}{E_{2}} ; a_{66}=\frac{1}{G_{12}} ; a_{16}=a_{26}=0
$$

The characteristic equation (Eq. (1)) has four complex roots. Two of them, $\mu_{1}$ and $\mu_{2}$ have positive imaginary part. The other two, $\mu_{3}$ and $\mu_{4}$, are their complex conjugates (i.e. $\mu_{3}=\bar{\mu}_{1}$ and $\mu_{4}=$ $\bar{\mu}_{2}$ ). With reference to the coordinates system $\left\{x_{1}, x_{2}\right\}$ centred on the crack tip (Figure 1), it is possible to define the complex variables $z_{1}$ and $z_{2}$ as:

$$
z_{j}=x_{1}+\mu_{j} x_{2} ; j=1,2
$$

and to obtain the displacements in terms of the arbitrary stress functions, $\Phi\left(z_{1}\right)$ and $\Psi\left(z_{2}\right)$ : 


$$
\begin{aligned}
& u_{1}=2 \Re\left\{p_{1} \Phi\left(z_{1}\right)+p_{2} \Psi\left(z_{2}\right)\right\} \\
& u_{2}=2 \Re\left\{q_{1} \Phi\left(z_{1}\right)+q_{2} \Psi\left(z_{2}\right)\right\}
\end{aligned}
$$

where $p_{j}, q_{j}(j=1,2)$ are the complex properties of the material defined as:

$$
\begin{gathered}
p_{j}=a_{11} \mu_{j}^{2}+a_{12}-a_{16} \mu_{j} \\
q_{j}=\frac{a_{12} \mu_{j}^{2}+a_{22}-a_{26} \mu_{j}}{\mu_{j}}
\end{gathered}
$$

Performing a conformal transformation from the physical plane, $z$, to the complex plane, $\zeta$ :

$$
z_{j}=-\zeta_{j}^{2} ; \quad \zeta_{j}=i \sqrt{Z_{j}}
$$

it is possible, following Lin et al. [20], to express the stress functions as power series:

$$
\begin{gathered}
\Phi\left(\zeta_{1}\right)=-\sum_{j=1}^{N} i\left(A_{j} \zeta_{1}^{j}\right) \\
\Psi\left(\zeta_{2}\right)=-\sum_{j=1}^{N} i\left(-\bar{A}_{j} B+A_{j} C\right) \zeta_{2}^{j}
\end{gathered}
$$

where $N$ is the number of terms of the power series, $i$ is the imaginary unit, and $B$ and $C$ are material properties defined as:

$$
\begin{gathered}
B=\frac{\bar{\mu}_{2}-\bar{\mu}_{1}}{\mu_{2}-\bar{\mu}_{2}} \\
C=\frac{\bar{\mu}_{2}-\mu_{1}}{\mu_{2}-\bar{\mu}_{2}}
\end{gathered}
$$

The coefficients $A_{j}$ are complex numbers, so it is possible to express them as $A_{j}=A_{R j}+i A_{I j}$, where the subscripts $A_{R j}$ and $A_{I j}$ denote the real and imaginary part of $A_{j}$, respectively. Substituting Eqs. (7) - (11) in Eqs. (3) and (4) it is possible to express the displacements as:

$$
\begin{aligned}
& u_{1}=2 \Re \sum_{j=1}^{N}\left\{-i A_{R j}\left[p_{1} \zeta_{1}^{j}+p_{2} \zeta_{2}^{j}(C-B)\right]+A_{I j}\left[p_{1} \zeta_{1}^{j}+p_{2} \zeta_{2}^{j}(C+B)\right]\right\} \\
& u_{2}=2 \Re \sum_{j=1}^{N}\left\{-i A_{R j}\left[q_{1} \zeta_{1}^{j}+q_{2} \zeta_{2}^{j}(C-B)\right]+A_{I j}\left[q_{1} \zeta_{1}^{j}+q_{2} \zeta_{2}^{j}(C+B)\right]\right\}
\end{aligned}
$$


Eqs $(12,13)$ are equivalent to the ones expressed in [21]. For the determination of the coefficients $A_{R j}$ and $A_{I j}$, it is sufficient to consider any linear combination of the displacements ( $\varphi=$ $c_{1} u_{1}+c_{2} u_{2}$ being $c_{1}$ and $c_{2}$ scalars), its value at the $k$-th macropixel, $\varphi_{k}$, and the corresponding experimental value, $\tilde{\varphi}_{k}$ (that is determined using the DIC). If this is done for $n$ points, the following system of linear equations is obtained:

$$
\left\{\begin{array}{c}
\tilde{\varphi}_{1} \\
\tilde{\varphi}_{2} \\
\vdots \\
\tilde{\varphi}_{n-1} \\
\tilde{\varphi}_{n}
\end{array}\right\}=\left\{\begin{array}{ccccc}
a_{R 11} & a_{I 11} & \cdots & a_{R 1 N} & a_{I 1 N} \\
a_{R 21} & a_{I 21} & \cdots & a_{R 2 N} & a_{I 2 N} \\
\vdots & \vdots & \cdots & \vdots & \vdots \\
a_{R N-11} & a_{I N-11} & \cdots & a_{R N-1 N} & a_{I N-1 N} \\
a_{R N 1} & a_{I N 1} & \cdots & a_{R N N} & a_{I N N}
\end{array}\right\}\left\{\begin{array}{c}
A_{R 1} \\
A_{I 1} \\
A_{R 2} \\
A_{I 2} \\
\vdots \\
A_{R N-1} \\
A_{I N-1} \\
A_{R N} \\
A_{I N}
\end{array}\right\}
$$

or, in compact form:

$$
\{\tilde{\varphi}\}=[a]\{A\}
$$

where $\{\tilde{\varphi}\}$ is the vector containing the experimental data, $[a]$ is a matrix containing the material parameters, and $\{A\}$ is the vector of the unknown coefficients that can be determined by using an over-deterministic least square fitting procedure.

The calculation of the coefficients of $\{A\}$ relies on the correct determination of the location of the crack tip. This is done implementing an optimisation methodology proposed by Pitarresi et al. [22] for the case of experimental data obtained from Thermoelastic Stress Analysis. The optimisation algorithm consists in finding the coordinate of the crack tip, $\left(\breve{x}_{1}, \breve{x}_{2}\right)$ that maximises the coefficient of determination $R^{2}\left(0 \leq R^{2} \leq 1\right)$ :

$$
R^{2}=1-\frac{R S S}{T S S}
$$

where the Residual Sum of Squares, RSS, and the Total Sum of Squares, TSS, are defined as:

$$
T S S=\sum_{k=1}^{n}\left(\tilde{\varphi}_{l}-\overline{\tilde{\varphi}}\right)^{2}
$$




$$
R S S=\sum_{k=1}^{n}\left(\tilde{\varphi}_{k}-\varphi_{k}\right)^{2}
$$

being $\overline{\tilde{\varphi}}=\sum \tilde{\varphi}_{k} / n$ the mean of the observed data. The guess domain over which the values of $R^{2}$ are iteratively evaluated consists in a small region around the expected location of the crack tip that is selected by the user at the beginning of the optimisation procedure. If the location of the crack tip is assumed to be at the centre of the subset, the optimisation procedure trivially calculates the values of $R^{2}$ at each analysed point in the selected Region of Interest (ROI), identifying the location of the crack-tip with the coordinates of the centroid $\left(x_{1}^{c}, x_{2}^{c}\right)$ of the subset for which the coefficient of determination takes its maximum value $R^{2} \sim 1$. This procedure allows the determination of the location of the crack tip with the accuracy of the chosen subset spacing, which in the best but most labour-intensive case may coincide with the native image the pixel resolution. If such accuracy is sufficient or not, then ultimately depends on the spatial resolution of ROI, i.e. on the subset spacing adopted. If an accuracy of the sub-ROI-spacing is desired, a further optimisation could be made, this time considering as a domain the region centred on the last pixel previously identified by the coarse optimisation (i.e. $x_{k} \in\left[x_{k}^{c}-\frac{s}{2}, x_{k}^{c}+\frac{s}{2}\right]$ for $k=1,2$, where $s$ is the side of the subset that is equal to 1 or $\frac{1}{f}$ depending on whether its dimensions are expressed in subset-spacings or mm). It should be noted that the choice of $\varphi$ is not completely arbitrary since it will affect the accuracy of the fitting, as it will be shown in the following. For the case of mode I, the SIF can be calculated from the first term of Eq. (13) as [23]:

$$
\mathcal{K}_{I}=-\sqrt{2 \pi} \mathfrak{R}\left(\frac{\mu_{1}-\mu_{2}}{\mu_{2}}\right) A_{R 1}
$$

\subsection{Procedure to evaluate the J-Integral}

Let $\left\{x_{1}, x_{2}\right\}$ be a Cartesian coordinate system with $x_{1}$ aligned with the crack growth direction (i.e. Figure 2). The J-integral for a two-dimensional body is calculated as [11]: 


$$
\mathcal{J}=\int_{\Gamma}\left(w d x_{2}-t_{j} \frac{\partial u_{j}}{\partial x_{1}} d s\right)
$$

where $\Gamma$ is an arbitrary path around the crack tip, $w$ is the strain energy density (calculated as $w=$ $\frac{1}{2} \sigma_{i j} \varepsilon_{i j}$ where $\sigma_{i j}$ and $\varepsilon_{i j}$ are the stress and strain tensors, respectively), and $t_{j}$ are the tractions along the path $\left(t_{j}=\sigma_{j i} n_{i}\right.$, where $\{n\}$ is the normal to $\Gamma$ and $n_{i}$ is the $i$-th component of $\left.\{n\}\right)$.

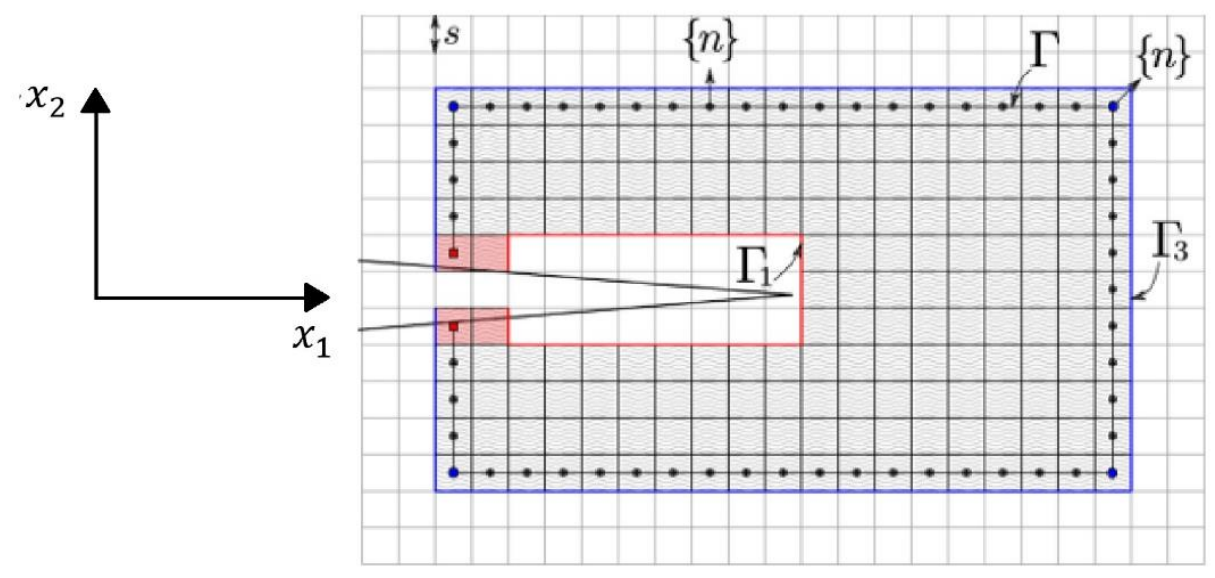

Figure 2 - Representation of the integration path $(\Gamma)$ and area (in grey) required for the numerical calculation of $\mathcal{J}$.

The contour integral of Eq. (19) allows the numerical calculation of the J-integral as done by Catalanotti et al. [18]. Here, in order to improve the accuracy of the method, we propose to calculate the J-Integral by using the formulation based on the Domain Integral Method. By applying the divergence theorem, after having defined an auxiliary function $Q$, Shih et al. [24] demonstrated that the J-integral of Eq. (19) can also be calculated as an area integral:

$$
\mathcal{J}=\int_{\Omega}\left[\left(\sigma_{l m} \frac{\partial u_{m}}{\partial x_{1}}-w \delta_{1 l}\right) \frac{\partial Q}{\partial x_{l}}\right] d A \quad l, m=1,2
$$

where $\Omega$ is the domain of integration (grey area in Figure 2), $Q$ is an arbitrary continuous smooth function that takes the value of 0 and 1 on the inner $\left(\Gamma_{1}\right)$ and outer boundary $\left(\Gamma_{3}\right)$, respectively, and $\delta_{l m}$ is the Kronecker delta $\left(\delta_{l m}=1\right.$ if $l=m$, and $\delta_{l m}=0$ if $\left.l \neq m\right)$.

Eq. (20) is expected to be more effective when calculating the J-integral by using field data, e.g. as those obtained by means of the DIC. To explain this assumption let us consider Figure 2 where the two cases, calculating the J-Integral using Eq. (19) or Eq. (20) are analysed. In Figure 2, the grid 
is made by all the subsets centred on the points where the displacement is evaluated by the DIC analysis.

If the J-Integral is calculated as in Eq. (19), the arbitrary path $\Gamma$ needs to connect the centroid of the subsets (Figure 2). It is convenient to choose a rectangular path in order to simplify the numerical calculation of the terms in Eq. (19) [18]. Although this methodology was successfully used to measure the J-Integral in orthotropic materials, care needs to be taken when choosing the parameters in order to ensure a sufficient level of accuracy.

One issue arises from the fact that the displacement is not available in the subsets located at the border of the specimen (dots in red in Figure 2). A second issue is that the normal to the boundary is not always easily calculated. This is the case of singular points, where the normal is simply assumed (point in blue in Figure 2). Finally, although the DIC is an experimental method able to provide the entire displacement field, only a small portion of the data available, that related to the subsets located on $\Gamma$, is used in Eq. (19). All these reasons may affect the accuracy of the method.

If Eq. (20) is used, the accuracy of the method is improved. In this case, in fact, the calculation of the J-Integral is done considering the information calculated in more points, i.e. all the subsets contained in $\Omega$ (grey area in Figure 2), making the method less affected by local errors. If the error related to the subsets located at the border of the specimen (in pink in Figure 2) is still maintained (this is an intrinsic issue related to the DIC and not to the equation used for the calculation of the JIntegral), its effect is mitigated by the fact that more subsets are considered. Finally, there is no need to calculate any normal.

The numerical calculation of Eq. (20) is done adapting a formulation proposed by Shih et al. [24] for the calculation of the J-Integral by using the finite element method. Hence, the J-Integral can be calculated as the sum of the contributions to the J-Integral given by each subset:

$$
\mathcal{J}=\frac{n A}{h} \sum_{k=1}^{n}\left[\left(\sigma_{l m} \frac{\Delta u_{m}}{\Delta x_{1}}-w \delta_{1 l}\right) \frac{\Delta Q}{\Delta x_{l}}\right]_{k}
$$


where $n$ is the number of subsets in $\Omega, A=s^{2}=1 / f^{2}$ is the area of each subset, $h$ is the thickness of the orthotropic body, $\sigma_{l m}$ are the stresses, $\frac{\Delta u_{m}}{\Delta x_{1}}$ is the numerical derivative of the displacement with respect to $x_{1}$, and $\frac{\Delta Q}{\Delta x_{l}}$ is the numerical derivative of $Q$ with respect to $x_{l}$.

Rearranging Eq. (21) as:

$$
\mathcal{J}=\frac{n}{h f^{2}} \sum_{k=1}^{n}\left[\left(\sigma_{l m} \frac{\Delta u_{m}}{\Delta x_{1}}-\frac{1}{2} \sigma_{l m} \varepsilon_{l m} \delta_{1 l}\right) \frac{\Delta Q}{\Delta x_{l}}\right]_{k}
$$

it can be observed that:

- the thickness of the specimen, $h$, the conversion factor, $f$, and the number of subsets, $n$, are known;

- $Q$ is imposed by the user, and its derivative, with respect to $x_{l}$, can be calculated numerically as the ratio $\Delta Q / \Delta x_{l}$;

- the displacements, $u_{m}$, and strains, $\varepsilon_{l m}$, are obtained from the DIC, and the numerical calculation of $\Delta u_{m} / \Delta x_{1}$ does not present particular difficulties;

- the stresses, $\sigma_{l m}$, are calculated knowing the stiffness matrix of the material, $\{\sigma\}=[C]\{\varepsilon\}$.

It should be observed that, since the material is considered to be linear elastic, the J-Integral allows the determination of both the energy release rate (ERR), $\mathcal{G}_{I}$, and the SIF, $\mathcal{K}_{I}$ [25]:

$$
\mathcal{J}=\mathcal{G}_{I}=\frac{\mathcal{K}_{I}^{2}}{\hat{E}}
$$

where $E$ is the equivalent Young's modulus defined as [26]:

$$
E=\left(a_{11} a_{22} \frac{1+\varrho}{2}\right)^{-\frac{1}{2}} \lambda^{\frac{1}{4}}
$$

being $\varrho$ and $\lambda$ two dimensionless material properties that take into account the orthotropy of the material:

$$
\varrho=\frac{2 a_{12}+a_{66}}{2 \sqrt{a_{11} a_{22}}} ; \quad \lambda=\frac{a_{11}}{a_{22}}
$$




\section{Experimental Work}

\subsection{Digital Image Correlation}

Digital Image Correlation (DIC) is an experimental technique which allows the evaluation of fullfield displacements in a material or structure by numerically correlating a set (at least two) of textured pattern images, each characterized by a given deformation stage. As highlighted by Pan et al. [27], the advantages in using 2D DIC are linked to its low requirements in terms of experimental setup, wide measurement range, and full-field and contactless characteristics. On the other hand, specimens are required to be flat and remain parallel to the sensor of the imaging system during the test. Therefore, only in-plane displacements can be measured. These characteristics make 2D DIC one of the most used experimental techniques when the evaluation of in-plane displacements is required [28].

DIC allows obtaining displacements and strains in a specimen using a relatively simple experimental setup (only a digital camera and the application of a pattern on the target surface of the specimen are required). It is achieved comparing a pair of images of the undeformed and deformed structure, in a specific area of the structure, called Region Of Interest (ROI). In the subset-based DIC approach, the principle of DIC is to track the transformation of a kernel region between the undeformed image (reference image) and the deformed image. A square subset of $\mathrm{N} \times \mathrm{N}$ pixel is typically used to find the location of the points in the deformed image. The dimension of the subsets affects both the accuracy and the spatial resolution of the technique [20,21]. The subset spacing, $s$, is the distance between adjacent subsets and defines the number of points effectively available in the defined ROI.

Displacements are found defining a correlation function that evaluates the similarity between the reference subset and the target subset. Pixel accuracy in the evaluation of the displacement is computed minimizing the correlation function between the reference and the deformed image, while sub-pixel accuracy is obtained using for instance iterative algorithms and interpolation (i.e. Newton- 
Raphson). Finally, strains are typically evaluated using a local polynomial fitting algorithm over an extension defining a virtual strain gauge (VSG).

\subsection{Specimen manufacturing}

The material system used in this work is unidirectional carbon fibre reinforced epoxy Hexcel IM7-8552 pre-impregnated plies, whose elastic properties are reported in Table 1. The plies were laid-up in a $\left[(90 / 0)_{4}\right]_{\mathrm{s}}$ configuration, in order to obtain a balanced cross-ply plate with a thickness of $2 \mathrm{~mm}$ (16 plies, each one having a thickness of $0.125 \mathrm{~mm})$.

Table 1-Elastic properties of Hexcel IM7-8552 plies [31]
\begin{tabular}{cc}
$E_{1}$ & $171.42 \mathrm{GPa}$ \\
$E_{2}=E_{3}$ & $9.08 \mathrm{GPa}$ \\
$G_{12}=G_{13}$ & $5.29 \mathrm{GPa}$ \\
$G_{23}$ & $3.97 \mathrm{GPa}$ \\
$v_{12}=v_{13}$ & 0.32 \\
\hline
\end{tabular}

Single Edge Notched Tension (SENT) specimens (Figure 3) were machined from the plate with a notch, having a length of $12 \mathrm{~mm}$ and radius of $0.9 \mathrm{~mm}$. An artificial crack (about $4.7 \mathrm{~mm}$ ) has then been made with a saw (having a thickness of $0.2 \mathrm{~mm}$ ) to obtain a value of $a_{0} / W$ equal to 0.56. The tested specimen had a value of $W=30 \mathrm{~mm}$ and $a_{0}=16.7 \mathrm{~mm}$ and a total length $\mathrm{L}=$ $200 \mathrm{~mm}$.

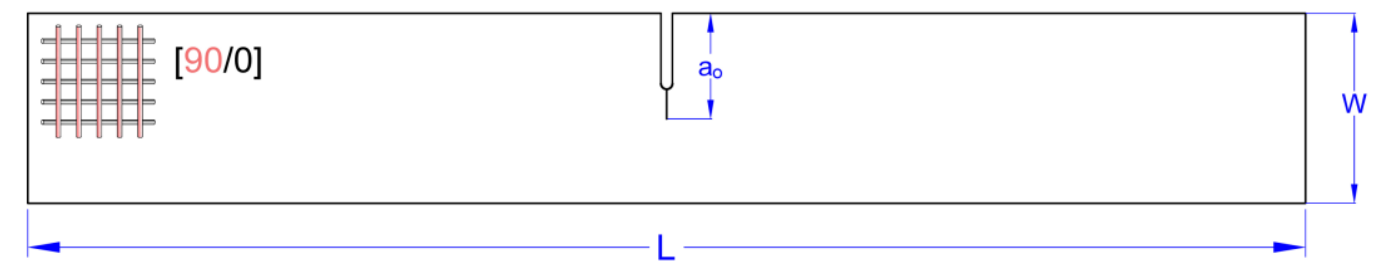

Figure 3-Sketch of the tested SENT specimens $a_{0}=15 \mathrm{~mm}, W=30 \mathrm{~mm}$.

Tests were carried out on a servo hydraulic INSTRON® 8801 universal test machine, at room temperature, under displacement control using a $100 \mathrm{kN}$ load cell, with a crosshead displacement of 
$0.2 \mathrm{~mm} / \mathrm{min}$. No tabs were applied on the specimen in the gripped area. Given the relatively low level of applied load at crack propagation, and the distance of the gripped area from the crack line, it was assumed that specimen tabbing was not necessary. It is assumed that the specimen is under plane stress conditions.

A 2D DIC setup was used. A 8-bit Baumer Optronic FWX20 Charged Coupled Device (CCD) camera coupled with a Nikon AF Micro-Nikkor $200 \mathrm{~mm}$ f/4D IF-ED lens was used for image grabbing. An acquisition frequency of $1 \mathrm{~Hz}$ was selected. The images were then processed using DIC MatchID software [32]. The DIC setting parameters were calibrated using the performance analysis tool. The converged parameters used in the analysis and post-processing are summarised in Table 2.

\begin{tabular}{lr}
\multicolumn{2}{c}{ Table 2-DIC setting parameters } \\
\hline Correlation Criterion & ZNSSD \\
Shape Function & Quadratic \\
Image Interpolation & Bicubic Spline \\
Image Filtering & Gaussian, with Kernel $5 \times 5$ \\
Subset Size & 10 pixels \\
Subset Step & 7 subsets \\
Strain Window & Bilinear Quadrilateral \\
Strain Interpolation & \\
\hline
\end{tabular}

The used ROI has a size of $185 \times 238$ subsets, with a conversion factor mm to pixels equal to $0.0097 \mathrm{~mm} /$ pixel. For the analysed specimen, 108 frames were available. Among the available frames, results are referred to the $30^{\text {th }}$ frame, which corresponds to an applied load of $4.6 \mathrm{kN}$. The Load-Displacement curve is shown in Figure 4 and the chosen load is highlighted. A representative contour plot for the component $u_{2}$ of the displacement is shown in Figure 5. 


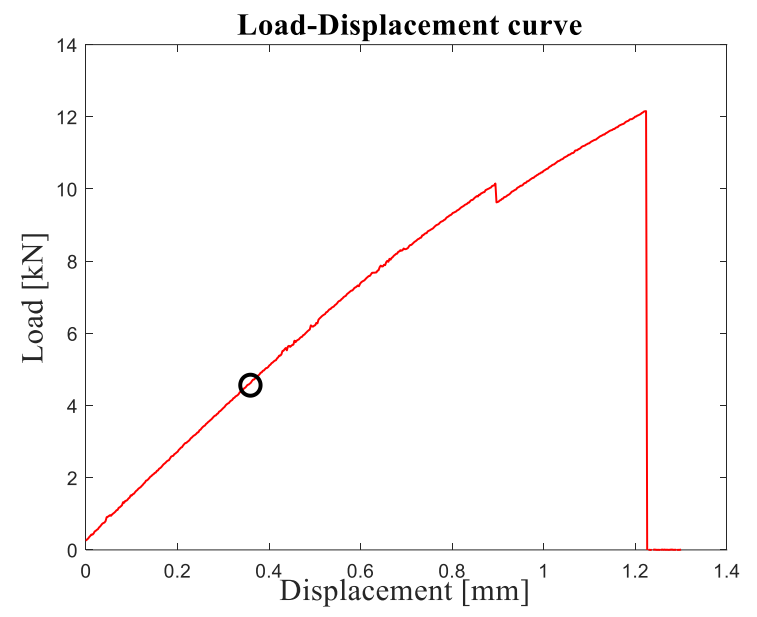

Figure 4-Load-Displacement curve of the tested specimen. The load corresponding to the chosen frame is highlighted.

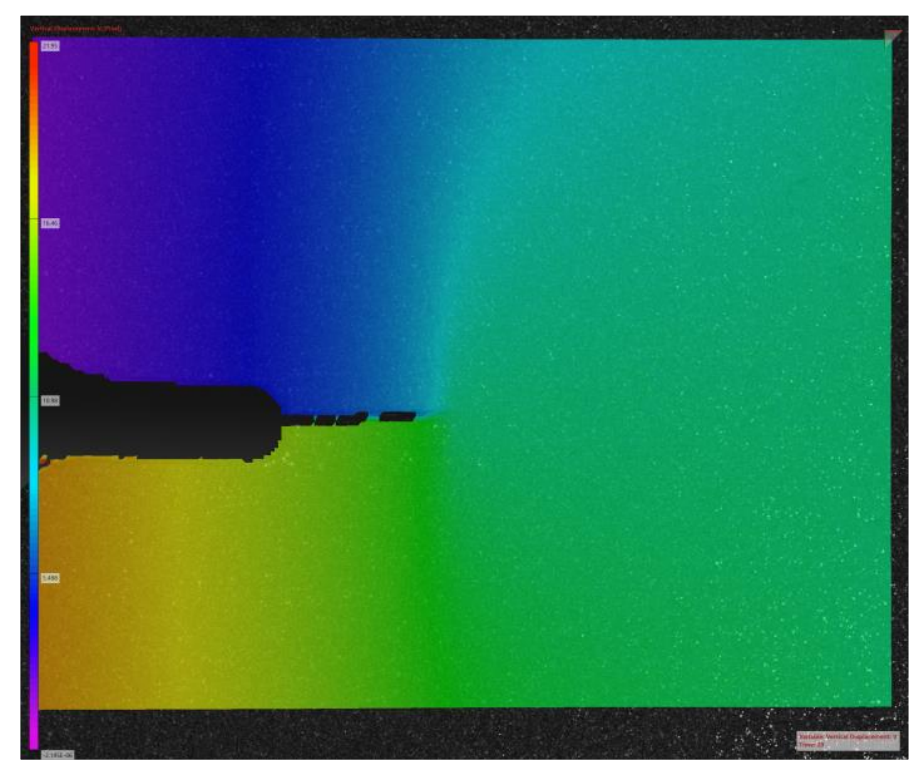

Figure 5 -contour plot of $u_{2}$ displacement at the $30^{\text {th }}$ frame.

\section{Experimental Results and Discussion}

\subsection{Determination of SIF and influence of parameters}

Both displacement components, $u_{1}$ and $u_{2}$, were considered when using the Over Deterministic Least Squares Fitting procedure for determining the SIF. As reported by Subramanyam et al. [2] this approach should lead to more stable and accurate results.

The displacements were fitted over an annular region centred on the crack tip (Figure 6) delimited by the inner and the outer radius $\left(r_{\min }\right.$ and $r_{\max }$, respectively) and by the angular 
coordinates $\left(\theta_{\max }\right.$ and $\left.\theta_{\min }\right)$, where the generic subset is identified referring to the polar coordinate system centred on the crack tip $\{r, \theta\}$. The use of these parameters allows excluding from the fitting the data that could undermine the accuracy of the fitting. In particular:

(i) it is necessary to define a $r_{\min }$ value in order to neglect points too close to the crack tip. In fact, since the region around the crack tip is characterised by the onset and propagation of damage, the data obtained in this region is not reliable. Moreover, the proposed fitting methodology assumes a linear elastic behaviour of the material, a condition that does not apply at the crack tip. Hence, the definition of a minimum radius allows the elimination of this problematic region from the fitting procedure;

(ii) if the fitting is performed on a region which is extremely large when compared to the region dominated by the SIF, the weight of the first term, $A_{R 1}$, decreases and this might affect the accuracy in the calculation of the SIF. Hence, it might be convenient in some cases to exclude the data that are too distant from the crack tip, and this could be done setting an appropriate value for $r_{\text {max }}$;

(iii)for the same reason, i.e. excluding data which could undermine the effectiveness of the fitting, it is possible to limit the angular extension of the region by defining the two angular coordinates $\theta_{\max }$ and $\theta_{\min }$ (in the following $\theta_{\max }=\theta_{\min }=\hat{\theta}$ ). The definition of $\hat{\theta}$ allows avoiding considering points with low significance as for example the points in proximity of the faces of the crack behind the crack tip. These points could also not provide reliable data since the DIC is unable to provide the displacement field in proximity of an edge. Subramanyam et al. [2] evaluated the influence of the angular extent, showing that angular values higher than $130^{\circ}$ allow to obtain more stable results. 


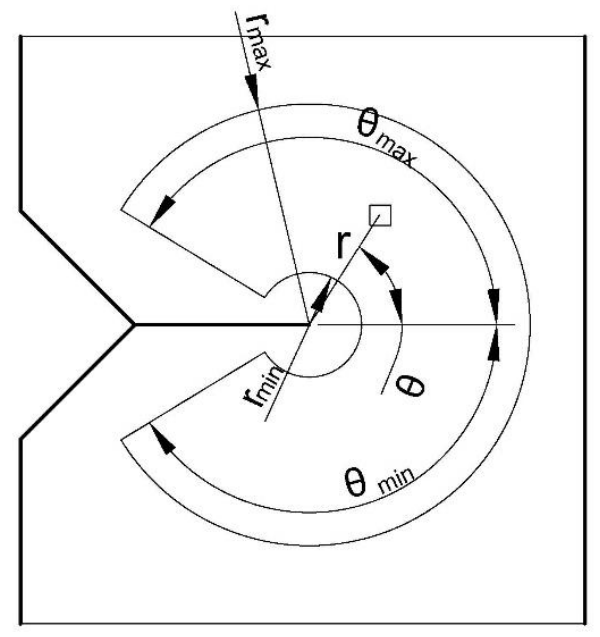

Figure 6 - Annulus sector area used in the fitting procedure

Since the formulation of the displacement used is a power series, also the effect of the number of terms has to be evaluated. The parameter used to quantify the quality of the fitting is the coefficient of determination, $R^{2}$, that is determined as in Equation (15). Since the specimen is subject to mode I loading, the displacements in the 1 direction, $u_{1}$, are much smaller than the displacements in the 2 direction, $u_{2}$. Hence, only the latter are used for this procedure. It should also be noted that although the annular region is considered for the fitting, the calculation of the coefficient of determination, $R^{2}$, is done considering the entire data available.

\subsubsection{Influence of $r_{\max }$ and $r_{\min }$}

Analyses were performed with an angular extension of the annular region of $\hat{\theta}=160^{\circ}$, and a number of Lekhnitskii's series terms of $N=4$. Keeping a constant value of $r_{\min }$ equal to $2 \mathrm{~mm}$, the value of $r_{\max }$ was changed between $5 \mathrm{~mm}$ and $15 \mathrm{~mm}$ (Figure 7). It is observed that $r_{\text {max }}$ has little influence on the SIF (Figure 7a), and virtually no influence on $R^{2}$ (Figure 7b). Hence, it is deduced that, for the case under investigation, it is not necessary to define a value of $r_{\text {max }}$. It can be postulated that this happens because the ROI contains points that are relevant for the calculation of the SIF. For this reason, in the following analyses a value of $r_{\max }$ is not defined.

Employing a similar procedure, the effect of $r_{\min }$ is calculated in the range from $1 \mathrm{~mm}$ to 10 $\mathrm{mm}$ (Figure 8). Although $r_{\min }$ does not affect the quality of the fitting (Figure $8 \mathrm{~b}$ ), it is noticed that 
the value of the SIF slightly decreases with increasing $r_{\min }$ (Figure 8a). This behaviour is expected because an increase of $r_{\min }$ corresponds to an exclusion of points belonging to the region dominated by the singularity. Hence, large values of $r_{\min }$ increases the weight of the non-singular terms while reduced values of $r_{\min }$ increase the weight of the singular term, which is the value from which depends the SIF. In the following $r_{\min }=2 \mathrm{~mm}$ was used.
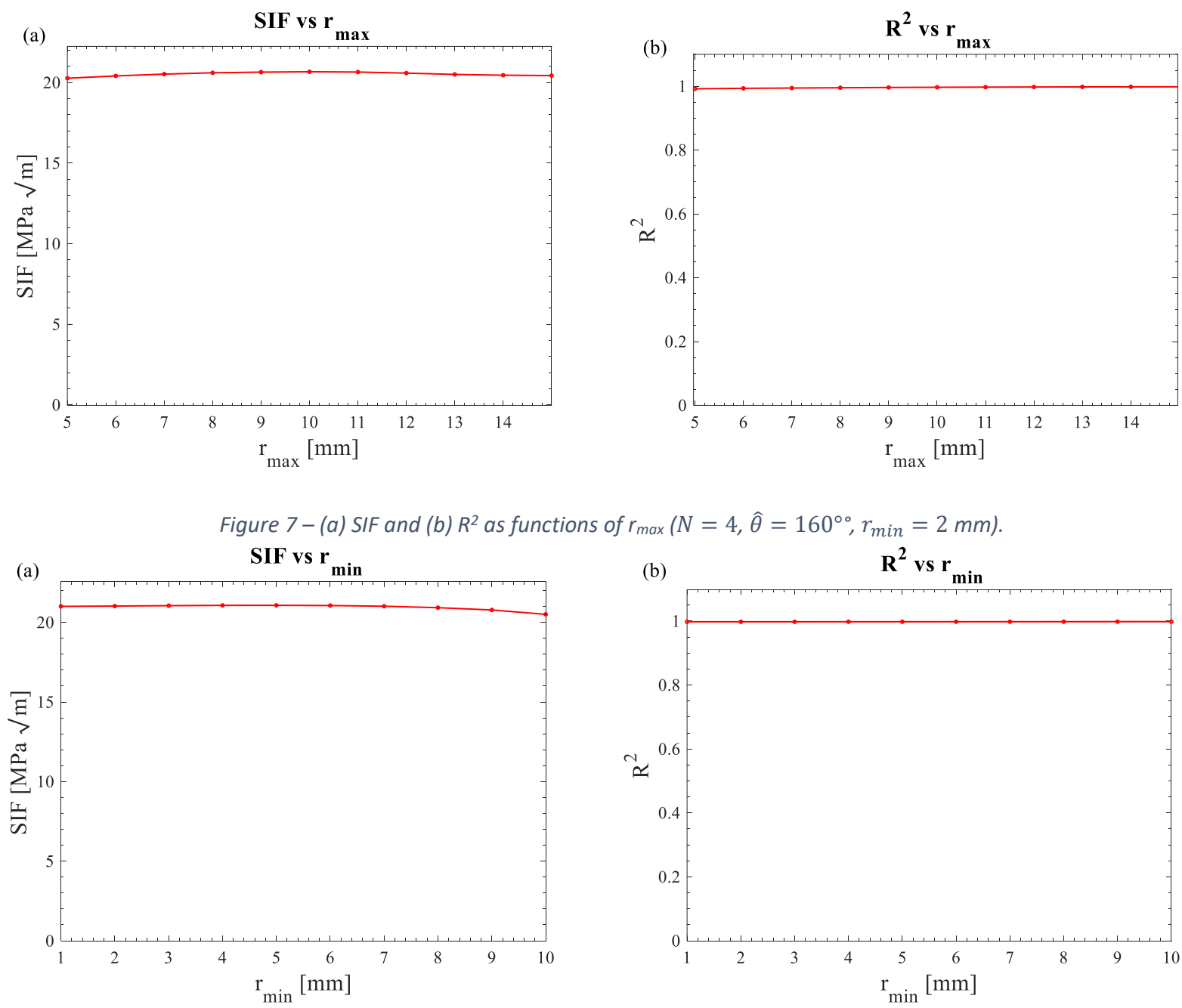

Figure 8 - (a) SIF and (b) $R^{2}$ as functions of $r_{\min }\left(N=4, \hat{\theta}=160^{\circ}, r_{\text {max }}\right.$ not defined).

\subsubsection{Influence of number of Lekhnitskii's series terms}

The effect of the number of the power series terms, $N$, was studied varying $N$ from 1 to 10

(Figure 9). The number of the power series terms does not seem to substantially affect the SIF which has an average values of $21 \mathrm{MPa} \sqrt{\mathrm{m}}$ and fluctuates between $20 \mathrm{MPa} \sqrt{\mathrm{m}}$ and $22 \mathrm{MPa} \sqrt{\mathrm{m}}$ (Figure 9a). 
Table 3 also reports and compares the values of the Lekhnitskii's displacement field coefficients, $A_{R J}$ and $A_{I J}$ (see also Eqs. 12-13) for the two cases of 4 and 10 coefficients truncation. It is in particular seen as the first coefficient, associated to the SIF is the biggest term, and the higher order coefficients becomes progressively smaller, indicating a decreasing weight of terms of $r$ with higher exponent order.

Table 3 - Comparison of Lekhnitskii's displacement field series coefficient

\begin{tabular}{|c|c|c|c|c|}
\hline \multicolumn{5}{|l|}{ Lekhnitskii's series } \\
\hline coefficient & $R e$ & $\mathrm{Im}$ & $R e$ & $I m$ \\
\hline$A_{1}\left[\mathrm{MPa} \times \mathrm{mm}^{1 / 2}\right]$ & -16.58 & -0.24 & -16.48 & 0.4 \\
\hline$A_{2}[\mathrm{MPa}]$ & -0.048 & -1.8 & -0.045 & -2.9 \\
\hline$A_{3}\left[\mathrm{MPa} \times \mathrm{mm}^{-1 / 2}\right]$ & 0.033 & 0.037 & 0.0079 & 0.08 \\
\hline$A_{4}[\mathrm{MPa} \times \mathrm{mm}]$ & -0.0001 & 0.0146 & 0.0024 & -0.016 \\
\hline$A_{5}\left[\mathrm{MPa} \times \mathrm{mm}^{-3 / 2}\right]$ & & & -0.00047 & 0.0019 \\
\hline$A_{6}\left[\mathrm{MPa} \times \mathrm{mm}^{-2}\right]$ & & & 0.00027 & 0.0022 \\
\hline$A_{7}\left[\mathrm{MPa} \times \mathrm{mm}^{-5 / 2}\right]$ & & & -0.00015 & 0.00025 \\
\hline$A_{8}\left[\mathrm{MPa} \times \mathrm{mm}^{-3}\right]$ & & & -0.00000143 & -0.00017 \\
\hline$A_{9}\left[\mathrm{MPa} \times \mathrm{mm}^{-7 / 2}\right]$ & & & 0.0000298 & 0.00000355 \\
\hline$A_{10}\left[\mathrm{MPa} \times \mathrm{mm}^{-4}\right]$ & & & -0.000000779 & 0.00000188 \\
\hline
\end{tabular}

The quality of the fitting is assessed calculating, separately, the values of the coefficient of determination, $R^{2}$, for each of the components of the displacement (i.e. $R^{2}\left(u_{1}\right)$ and $R^{2}\left(u_{2}\right)$ ). It is observed that while $R^{2}\left(u_{2}\right)$ does not virtually change with the number of Lekhnitskii's series terms $\left(R^{2}\left(u_{2}\right) \approx 1\right), R^{2}\left(u_{1}\right)$ increases noticeably, and reaches a plateau $\left(R^{2}\left(u_{1}\right) \approx 0.9\right)$ only for $N \geq 4$ (Figure 9b). 

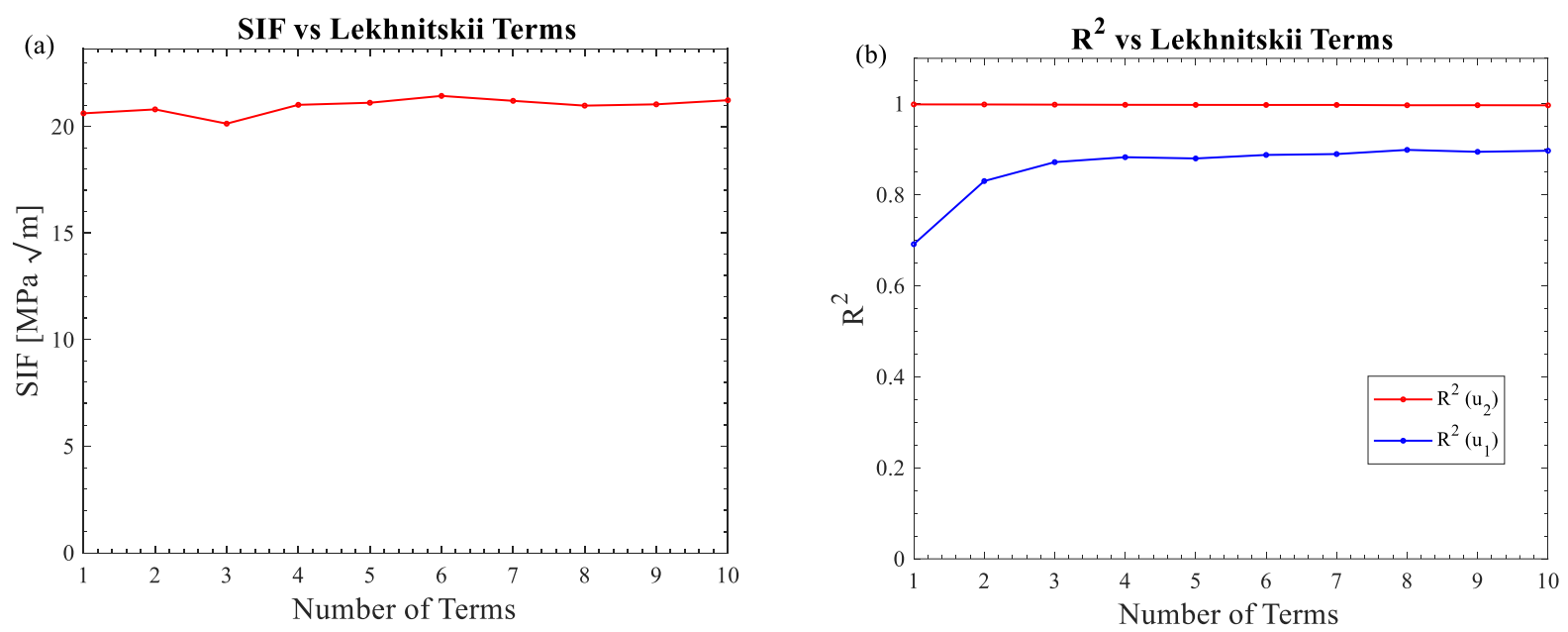

Figure 9-(a) Stress Intensity Factor and (b) $R^{2}$ as functions of the number of Lekhnitskii's series terms, $N\left(\hat{\theta}=160^{\circ}, r_{\text {min }}=2 \mathrm{~mm}\right.$, $r_{\text {max }}$ not defined).

Although $R^{2}$ increases with $N$ (mainly that related to the $u_{1}$ component), the best fit is obtained with a low number of terms $(N=4)$ as it is deduced observing the contour plots (Figure 10) that report, for different number of terms, the experimental and fitted displacement fields.
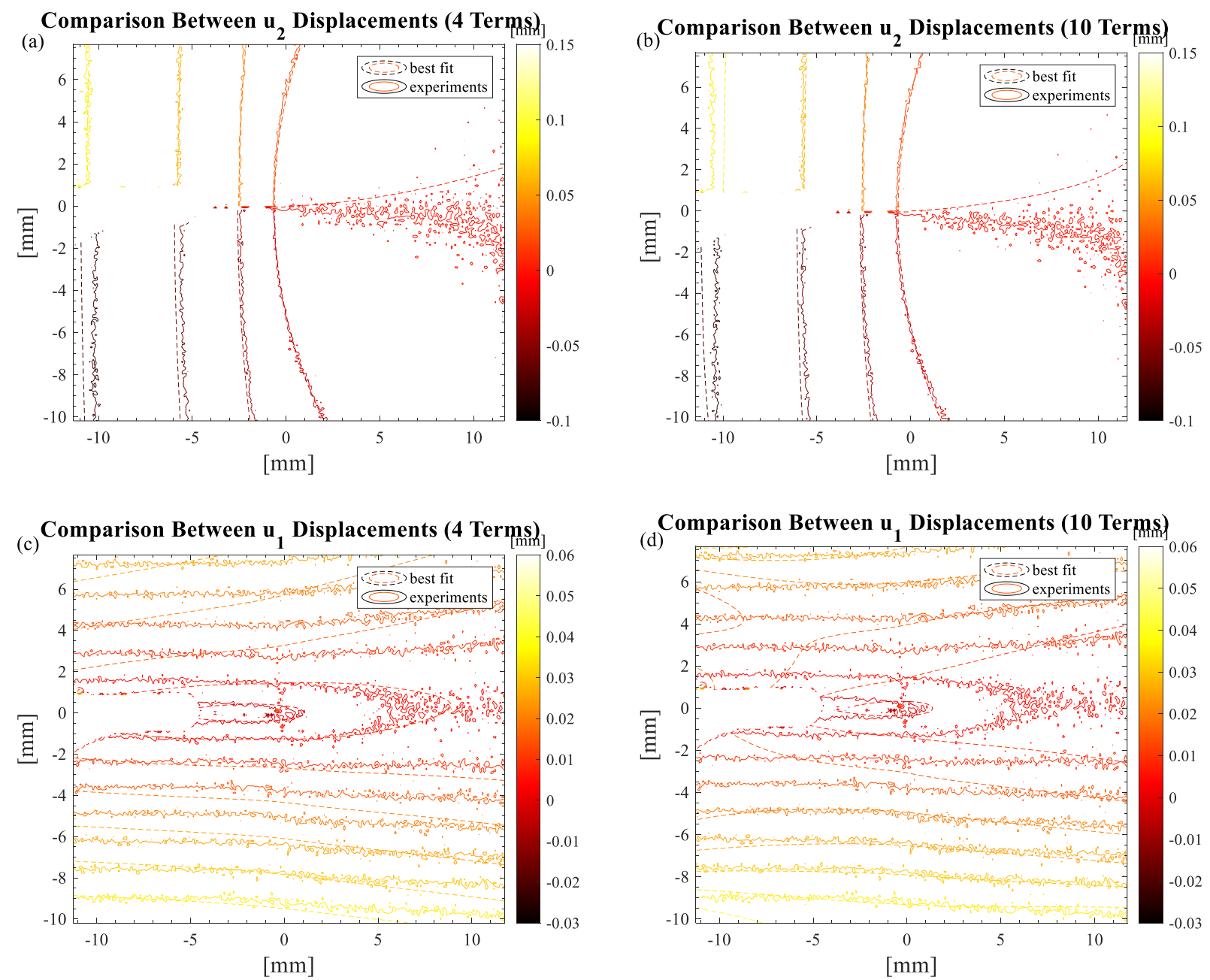

Figure 10 - Contour plot of the experimental and analytical method for both displacement fields, varying the number of terms: (a) $u_{2}$ when $N=4$, (b) $u_{2}$ when $N=10$, (c) $u_{1}$ when $N=4$, (d) $u_{1}$ when $N=10$. 
For a number of Lekhnitskii's series terms equal to $N=4$, the fitting is excellent (Figure 11), and the absolute error ${ }^{\dagger}$ contained (Figure 12).
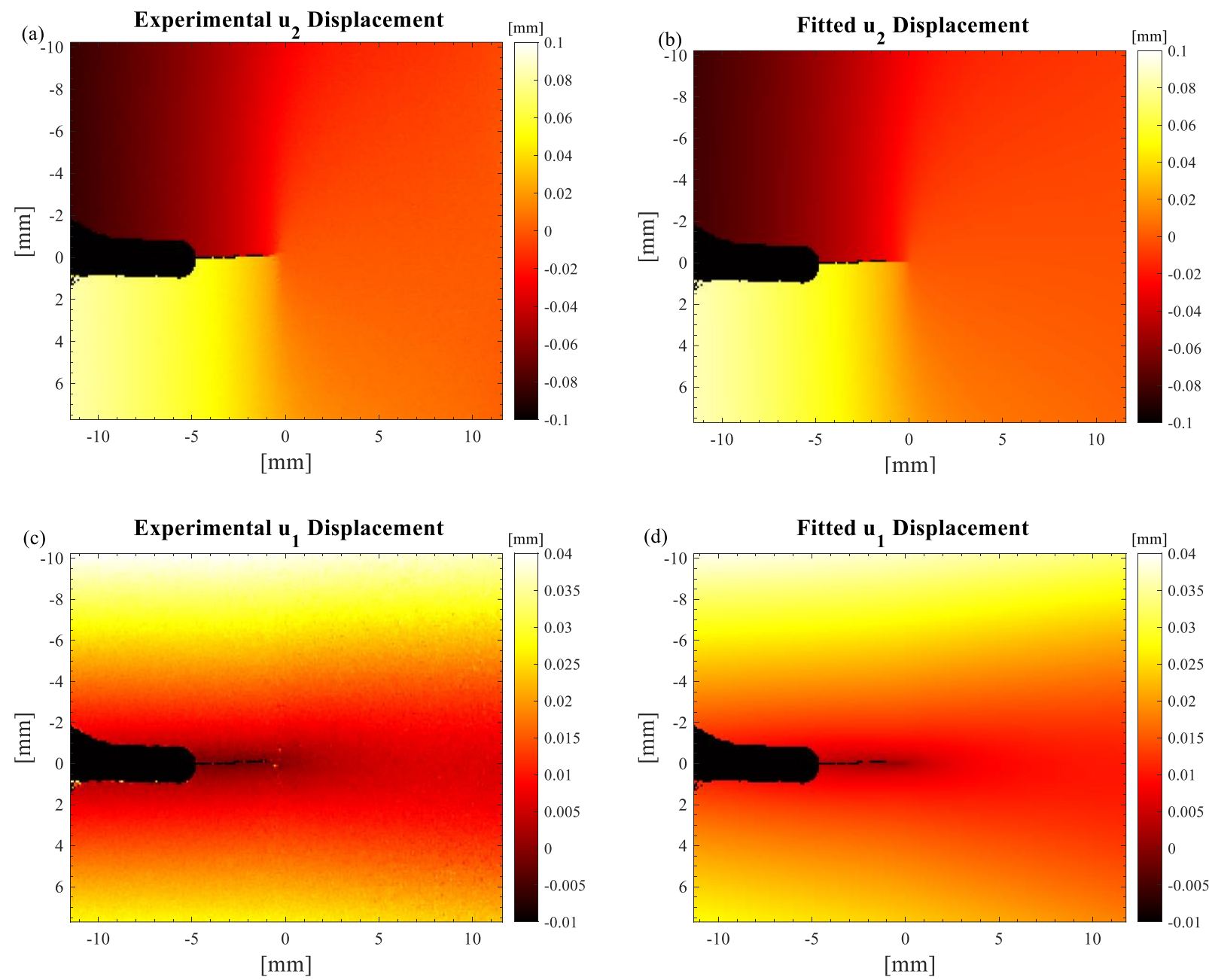

Figure 11 - Comparison between the (a) experimental and (b) fitted $u_{2}$ displacement and (c) experimental and (d) fitted $u_{1}$ displacement.

${ }^{+}$Calculated as the difference between the experimental and fitted values of the displacement field. 

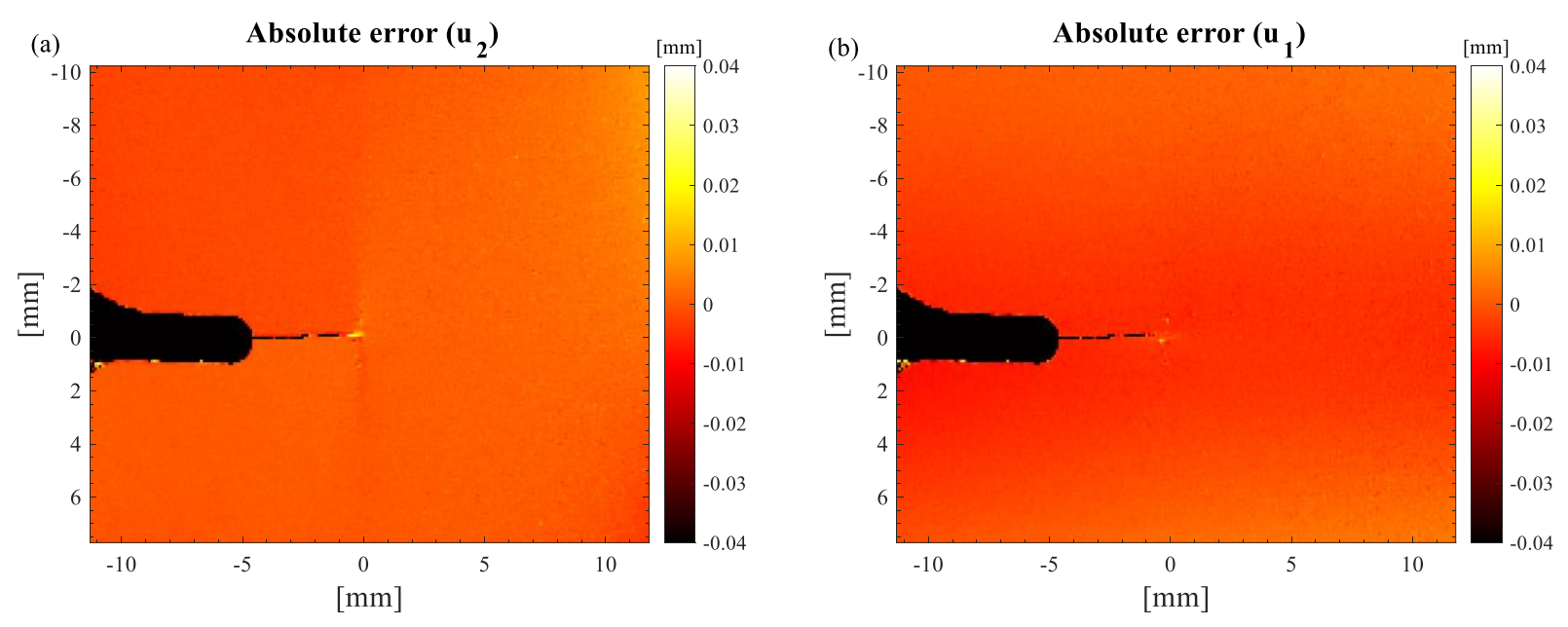

Figure 12 - Absolute error of both (a) $u_{2}$ and (b) $u_{1}$ displacements.

\subsection{Evaluation of J-Integral and influence of parameters}

The calculation of the J-Integral using the formulation of Equation (22) requires the definition of an integration domain (shaded area in Figure 13) that is delimited by two boundaries $\left(\Gamma_{1}\right.$ and $\left.\Gamma_{3}\right)$ as shown in Figure 2. In the following $\Gamma_{1}$ and $\Gamma_{3}$ are considered to be squares with side having $N_{\text {int }}$ and $N_{\text {ext }}$ subsets, respectively (Figure 13).

In an analogous way as previously done, $N_{\text {int }}$ and $N_{\text {ext }}$ can be used to exclude points that could affect the accuracy of the J-Integral calculation. The first parameter, $N_{\text {int }}$, allows to neglect points in the vicinity of the crack tip that need to be excluded because, as previously explained, are not able to provide reliable data for the displacement and strain field near geometric singularities at the notch and crack tip. The latter, $N_{\text {ext }}$, allows eliminating points near the edge for which the DIC is not able to provide an accurate value of the displacement.

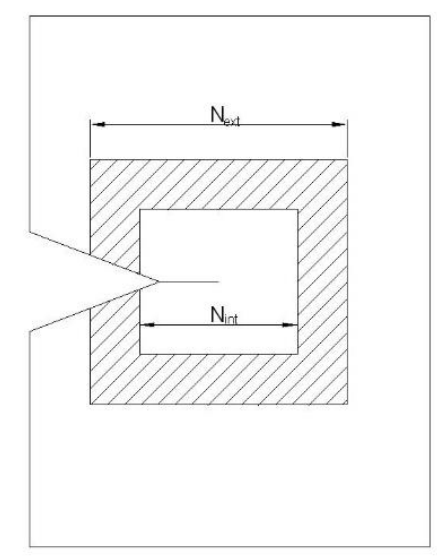


Several aspects need to be considered to assess the effectiveness of the proposed approach, and in particular:

(i) the ability of the method to provide a value of $\mathcal{J}$ that converges for increasing number of points in the integration domain;

(ii) the effect of the smoothing of the raw displacement field data;

(iii) the appropriate choice of the auxiliary function $Q$;

(iv) the sensitivity of the method.

\subsubsection{Convergence of the J-Integral}

The path independency of the J-Integral implies that, changing the integration domain, the calculated value of $\mathcal{J}$ should be constant; however, when a numerical approach is used, as in this case, the estimation is affected by an error that should decrease increasing the extension of the integration domain (the number of subsets, $n$ in Equation (22)). Hence, an increase of $n$ should cause the JIntegral to converge to a plateau value that approaches to the actual experimental value.

In order to perform this study, the J-Integral is calculated freezing the inner boundary (i.e. keeping constant the size of the inner boundary imposing $N_{\text {int }}=20$ ) and increasing the size of the outer boundary (i.e. increasing $N_{\text {ext }}$ ). It is observed that the proposed approach behaves as expected, providing a plateau value of $\mathcal{J}=11.8 \mathrm{~N} / \mathrm{mm}$ once a threshold value of $N_{\text {ext }}$ has been reached (purple curve in Figure 14). The methodology is fast and can be implemented on-the-fly, providing the JIntegral curves as a function of $N_{\text {ext }}$ for different values of the applied load (Figure 14). 


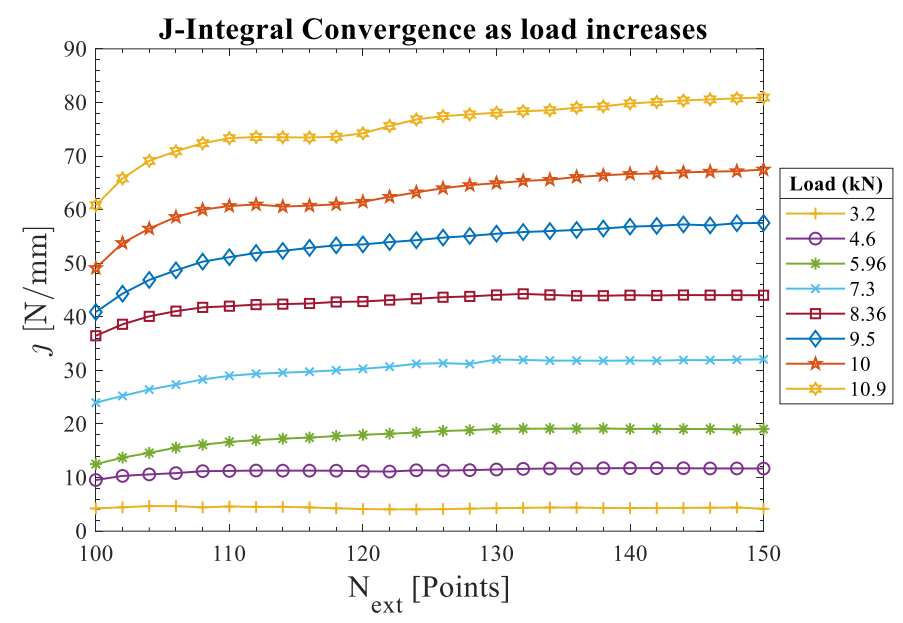

Figure 14 - J-integral as a function of $N_{\text {ext }}$ for different applied loads.

The ability of the proposed approach to converge to a plateau value of the J-Integral can be demonstrated also calculating $\mathcal{J}$ for different combinations of $N_{\text {int }}$ and $N_{\text {ext }}$.

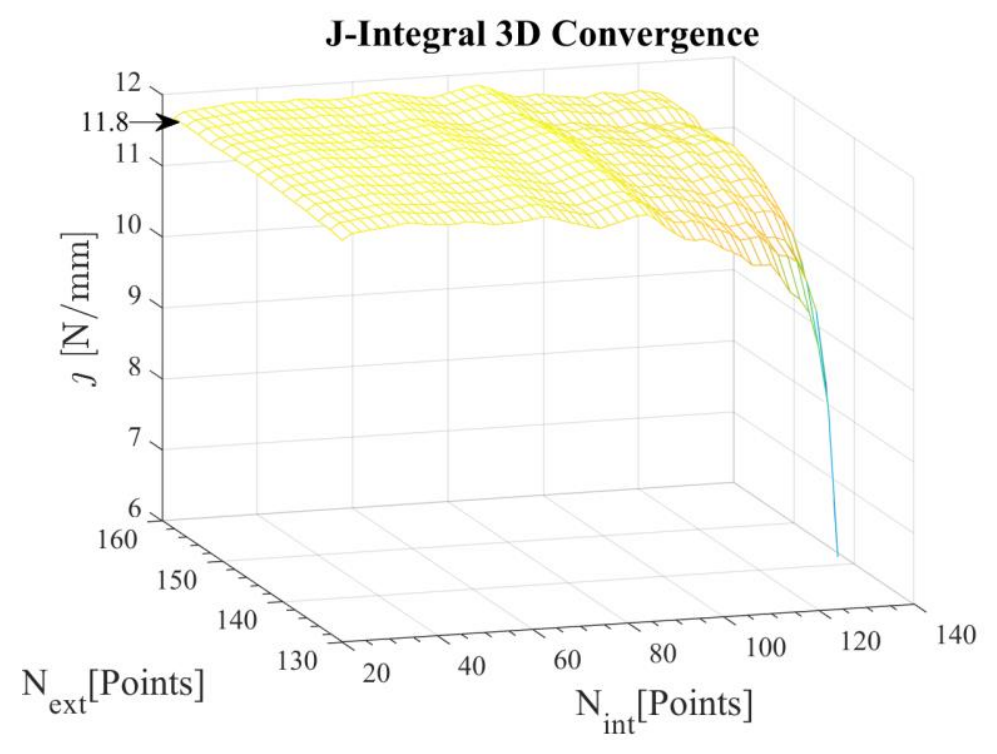

Figure 15 - Plateau value of the J-integral for different values of $N_{\text {int }}$ and $N_{\text {ext }}$.

With reference to the same case analysed earlier ( $30^{\text {th }}$ frame), it is possible to calculate the value of the J-Integral for $N_{\text {int }}$ and $N_{\text {ext }}$ in the range [20,124] and [130,150], respectively (Figure 15). It is observed that the plateau value of $\mathcal{J}$ is not correctly computed when both large values of $N_{\text {int }}$ and small values of $N_{\text {ext }}$ are simultaneously used. The reached plateau value is indicated by the arrow. 
Finally, the sensitivity of the method towards number of points included in the calculation is investigated. This has been performed varying both the dimensions of the inner and the outer contour. The error has been evaluated as the variation of $\mathcal{J}$ divided by the most accurate value.

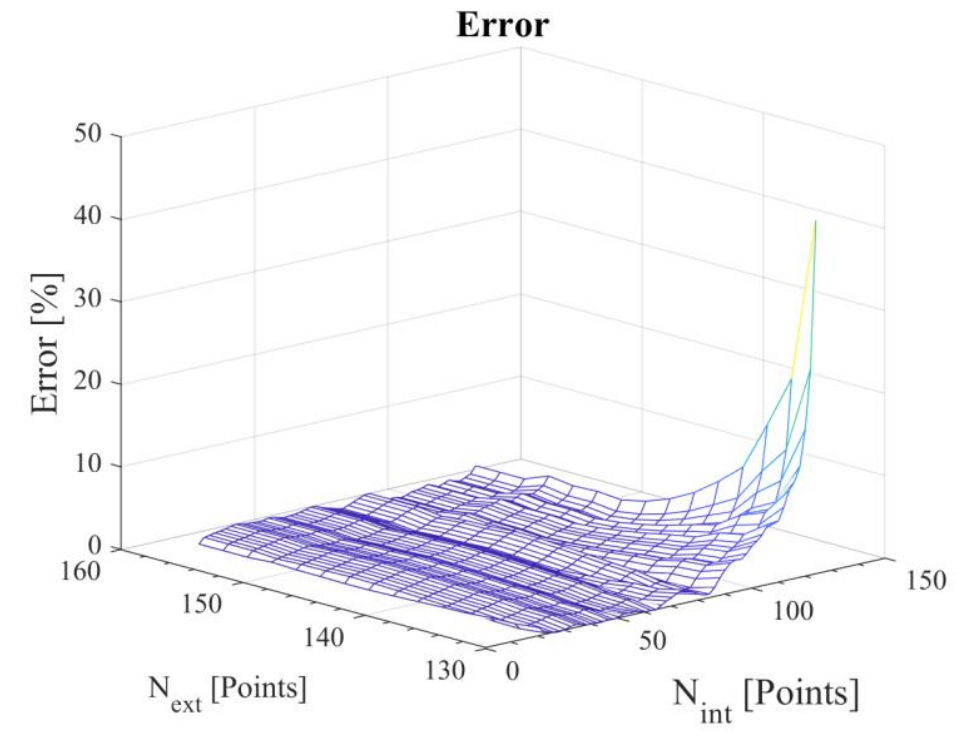

Figure 16 - Error as the number of points included in the calculation domain varies.

It is fair to assume that the most accurate value is the one obtained using in the calculation the highest number of experimental points. Since $N_{\text {int }}$ varies from 20 to 124 points while $N_{\text {ext }}$ varies from 130 to 150 points, the best value is considered the one obtained posing $N_{\text {int }}=20$ and $N_{\text {ext }}=$ $150(\mathcal{J}=11.8 \mathrm{~N} / \mathrm{mm})$. In Figure 16 it is shown the error (expressed in percentage terms). For most of the points, the error is close to 0 , as a confirm of the high stability of the developed method.

\subsubsection{Smoothing of the raw data}

The noise in the displacement raw data obtained from the DIC could significantly affect the calculation of the derivatives of the displacement ${ }^{+}$necessary for the calculation of $\mathcal{J}$ (Equation (22)), and could consequently cause a loss of accuracy of the proposed methodology. In order to improve the calculation of the derivative, it is possible to fit the experimental data using an analytical

\footnotetext{
${ }^{\ddagger}$ Equation (22) requires the calculation of $\Delta u_{m} / \Delta x_{1}$, that corresponds to the calculation of both $\Delta u_{1} / \Delta x_{1}$ and $\Delta u_{2} / \Delta x_{1}$. The first derivative, $\Delta u_{1} / \Delta x_{1}$, is equal to the strain, $\varepsilon_{11}$, whose calculation does not present any particular difficulty since it is directly provided by the DIC. On the contrary, the derivative, $\Delta u_{2} / \Delta x_{1}$, needs to be numerically calculated using the displacement field provided by the DIC.
} 
formulation. Although any analytical expression could be used, it is appropriate to fit the experimental data using the formulation reported in Equations (12) and (13).

This considerably improves the accuracy in the calculation of the derivative and provides a value of the $\mathrm{J}$-Integral equal to $\mathcal{J}=11.5 \mathrm{~N} / \mathrm{mm}$. This is due to the high reduction of the noise, in both the displacements and their derivatives, as shown in Figure 17.

In Figure 17c,d it is shown the derivative of the displacement component $u_{2}$ with respect to the $x_{2}$ direction. Even though the calculation of this derivative is not required in the evaluation of $\mathcal{J}$, it has been chosen to use it as an example of the noise-filtering effectiveness of the fitting algorithms.

(a)
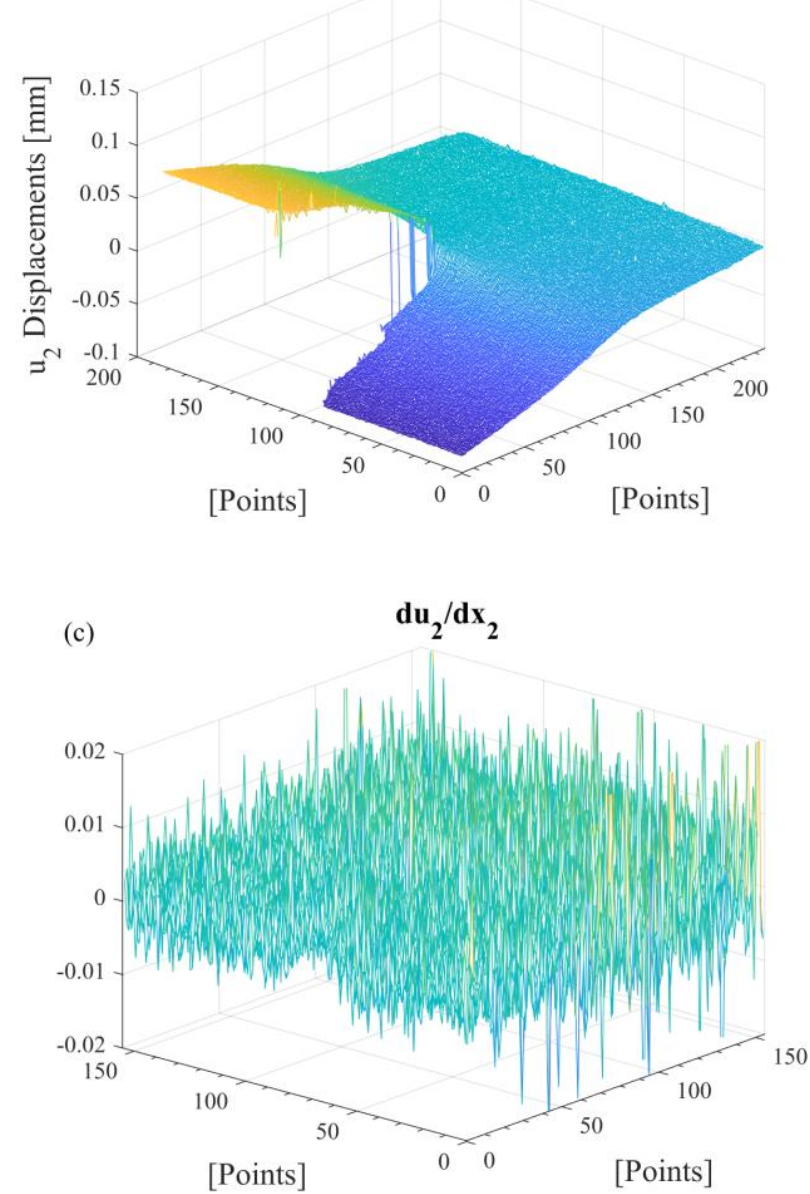

(b) $u_{2}$ Fitted (Smooth)
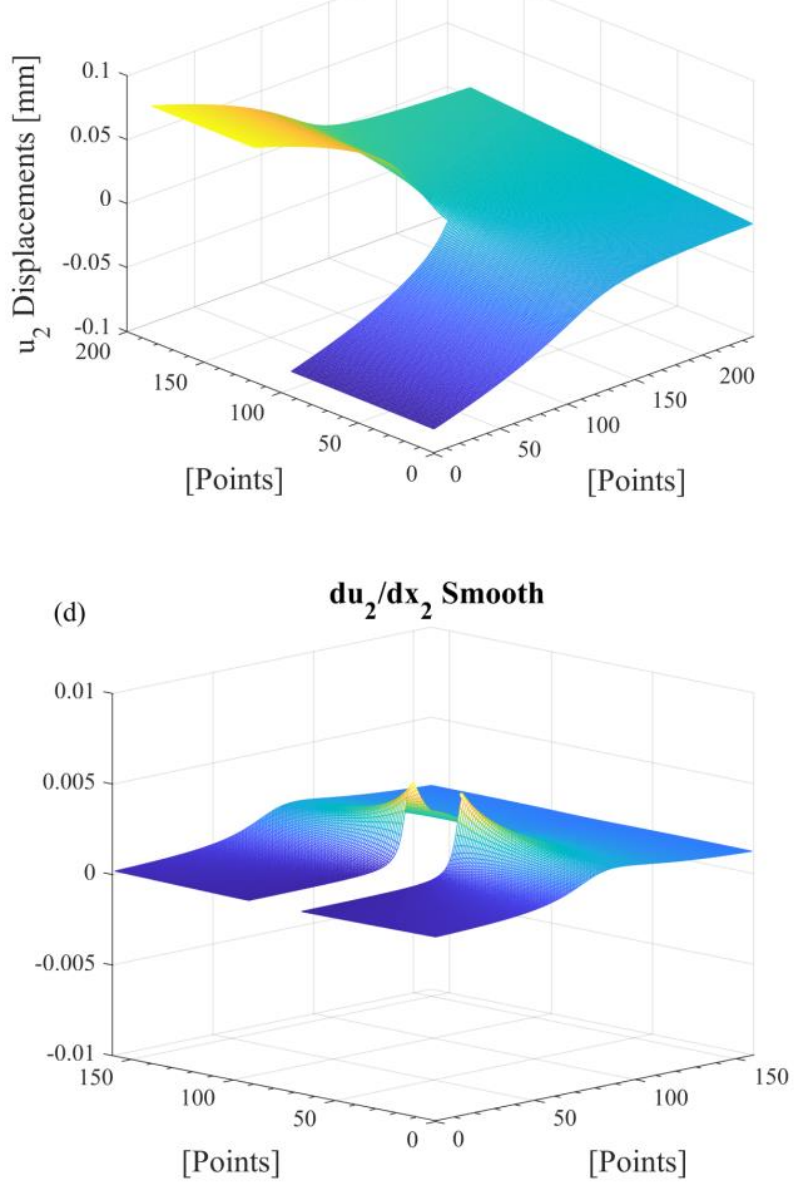

Figure 17 - Comparison between the (a) experimental and (b) fitted $u_{2}$ field, and their derivatives (c) experimental and (d) fitted with respect to the $x_{2}$ direction 


\subsubsection{Choice of the auxiliary function $Q$}

The auxiliary function $Q$ can be arbitrary but has to meet some requirements [24], and in particular (i) it has to be continuous, (ii) it has to take the values 0 and 1 in the outer and inner boundaries, respectively, and (iii) it has to take values in the range $[0,1]$ within the domain of integration.

Following Shih et al. [24], two analytical formulations are investigated for $Q$, and in particular:

(i) the pyramidal function, where $Q$ varies linearly in all the directions, from the inner boundary $\left(Q=1\right.$ at $\left.\Gamma_{1}\right)$ to the outer boundary $\left(Q=0\right.$ at $\left.\Gamma_{3}\right)$;

(ii) the plateau function, where $Q=1$ everywhere in $\Omega$ but $Q=0$ at the outer boundary $\left(\Gamma_{3}\right)$.

Both functions can be implemented in a Matlab [33] script (Figure 18) and allow the calculation of the J-Integral using Equation (22).

From a convergence study performed using both functions (Figure 19), it is observed that the use of the pyramidal or plateau functions is not equivalent and leads to different results. In fact, while the pyramidal function quickly converges to a limiting value of $\mathcal{J}$, the plateau function provides values that fluctuate around the same value (Figure 19a).
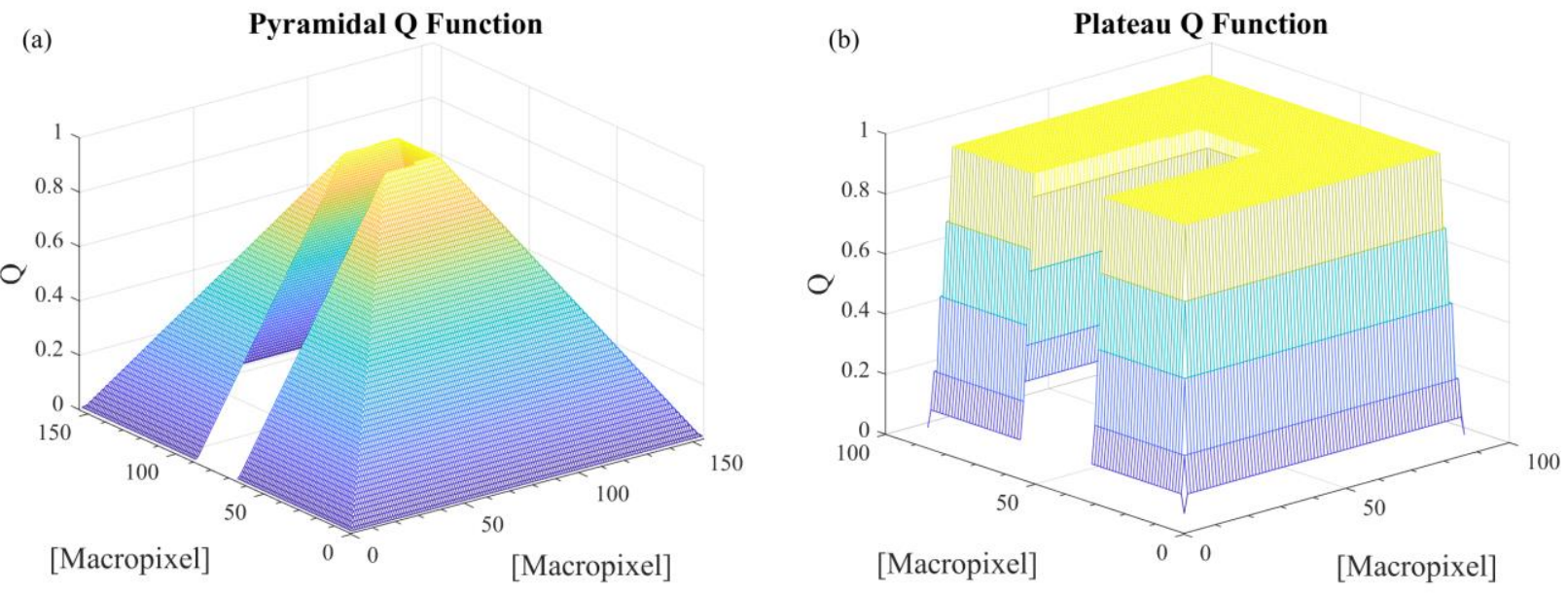

Figure 18 - (a) pyramidal and (b) plateau $Q$ functions. Missing points are due to the presence of the notch. 
This could be explained observing that in Equation (22) the derivatives $\Delta Q / \Delta x_{l}$ need to be calculated. In the case of the plateau function, these derivatives are 0 everywhere but on the subsets attached to the external boundary. This corresponds to the fact of considering, in the calculation of $\mathcal{J}$, only the contribution of the subset attached to the external boundary and neglecting that of the inner subsets. This fact partially negates the advantage of using an integral domain approach and makes the calculation sensibly affected by local experimental errors.

If the pyramidal function is used, the contribution of all the subsets in the integration domain is taken into account (i.e. the derivative is never zero). This makes the calculation less sensible to local errors and the convergence of the method more stable.

To further corroborate this hypothesis, the same calculation is repeated using the displacement field obtained by fitting the experimental data with the analytical formulation reported in Equations (12) and (13).

It is evident that in this case, the fitting eliminates the local errors, and this fact leads to a stable convergence when using both auxiliary functions. They both provide virtually the same limiting value (Figure 19b). The results obtained are summarised in Table 4.
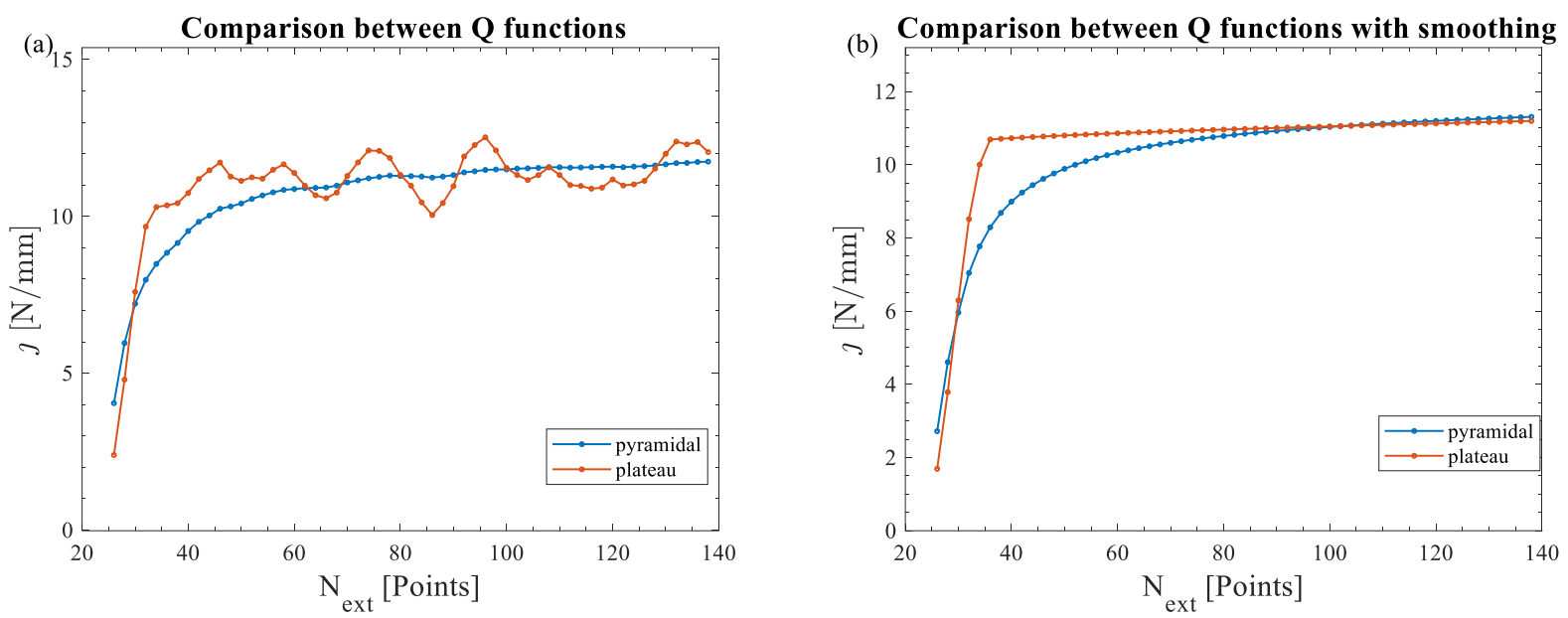

Figure 19 - Convergence comparison using pyramidal and plateau $Q$ functions (a) without and (b) with smoothing of the displacement field. 
Table 4-J-integral values obtained.

\begin{tabular}{lc}
\hline \multicolumn{1}{c}{ Smoothing on the displacement field } & $\mathcal{J}[\mathrm{N} / \mathrm{mm}]$ \\
\hline No smoothing applied to the displacement field & 11.8 \\
Smoothing applied to the displacement field & 11.5 \\
\hline
\end{tabular}

\subsection{Evaluation of SIF from J-integral and comparison}

The fitting procedure in 4.1 allows obtaining a value of the Stress Intensity Factor equal to 21.04 $\mathrm{MPa} \sqrt{\mathrm{m}}$. Assuming that the material has a linear-elastic behaviour, it is also possible to obtain SIF from $\mathcal{J}$, as shown in Eq. (28).

Table 5 -SIF values obtained by the different presented approaches.

\begin{tabular}{lc}
\hline \multicolumn{1}{c}{ Methods used to evaluate the mode I SIF } & $\mathcal{K}_{I}[\mathrm{MPa} \sqrt{\mathrm{m}}]$ \\
\hline SIF obtained from the fitting procedure & 21.04 \\
SIF obtained from J-integral & 22.12 \\
SIF obtained from J-integral, using fitted displacement data & 21.85 \\
Numerically evaluated SIF & 20.34 \\
\hline
\end{tabular}

Table 5 summarises the values of SIF that can be obtained using different approaches. The three described experimental approaches are compared with a reference value obtained following the approach described by Catalanotti et al. [34,35]. Since the experimental values are fairly similar to each other and in good agreement with the reference value, this allows to verify the validity of the adopted techniques.

\section{Conclusions}

In this work a Single Edge Notched Tension sample, made of a CFRP IM7/8552 laminate with orthotropic behaviour, is tested for Mode I fracture. Two procedures are presented for the evaluation of the SIF, using the displacement field obtained with 2d Digital Image Correlation: a least 
square fitting procedure based on the Lekhnitskii's formulation, and a procedure based on the evaluation of the J-Integral as a domain integral.

The outcomes of this investigation are summarised in the following points:

i) The calculation of the SIF by fitting the displacements using Lekhnitskii's formulation requires care in choosing the region of interest. On one hand, particular care needs to be taken when choosing $r_{\min }$ which needs to be small enough to properly predict the singular term, and therefore the SIF. On the other hand, $r_{\min }$ should be large enough to exclude the fractured region from the fitting procedure. On the contrary, it was demonstrated that the effect of $r_{\max }$ is not significant. In this study, $r_{\min }=2 \mathrm{~mm}$ has been used and, in this particular case, $r_{\max }$ was not defined.

ii) A compromise needs to be found on the optimal number of terms of the power series needed. If an increase of $N$ improves the quality of the fitting, the number of terms need to be limited in order to avoid a badly conditioned fitting. In this study, a number of terms $N=4$ was used.

iii) In order to ensure the accuracy of the fitting, it is essential to correctly determine the location of the crack tip. In this study this was achieved through a simple iterative method.

iv) The methodology developed for the calculation of $\mathcal{J}$ has been proved to be robust and rapidly converging when increasing the domain of integration.

v) The use of a plateau or pyramidal expression for the auxiliary function, $Q$, does not lead to equivalent results especially when $\mathcal{J}$ is calculated directly from the raw DIC data (i.e. with no fitting). In this case, in fact, the use of a pyramidal function is preferable, since it allows considering in the calculation a larger number of experimental points thus reducing the effect of local errors and, consequently, leading to more stable results.

vi) The determination of $\mathcal{J}$ requires the calculation of the derivatives $\Delta u_{m} / \Delta x_{1}$, whose calculation, performed on scattered raw DIC data, provides poor results. To remedy this 
situation, these derivatives were calculated on the fitted displacements with improved accuracy.

vii) Since a good agreement was found when comparing the SIF calculated through the proposed methods, it can be concluded that these approaches provide equivalent results if the experimental parameters are carefully selected.

\section{Acknowledgements}

The first author would also like to acknowledge the financial support provided by the University of Palermo through the Erasmus + for traineeship 2018-19 mobility grant. Authors acknowledge Fundação para a Ciência e a Tecnologia (FCT - MCTES) for its financial support via the project UIDB/00667/2020 (UNIDEMI).

\section{References}

[1] H.. Sutton, M. A.; Orteu, J.J; Schreier, Image Correlation for Shape, Motion and Deformation Measurements, 1st ed., Springer US, 2009. https://doi.org/10.1007/978-0-387-78747-3.

[2] M. Subramanyam Reddy, K. Ramesh, A. Thiyagarajan, Evaluation of mode-I SIF, T-stress and Jintegral using displacement data from digital image correlation - Revisited, Theor. Appl. Fract. Mech. 96 (2018) 146-159. https://doi.org/10.1016/j.tafmec.2018.04.006.

[3] J.R. Yates, M. Zanganeh, Y.H. Tai, Quantifying crack tip displacement fields with DIC, Eng. Fract. Mech. 77 (2010) 2063-2076. https://doi.org/10.1016/j.engfracmech.2010.03.025.

[4] R. Harilal, C.P. Vyasarayani, M. Ramji, A linear least squares approach for evaluation of crack tip stress field parameters using DIC, Opt. Lasers Eng. 75 (2015) 95-102. https://doi.org/10.1016/j.optlaseng.2015.07.004.

[5] M. Zanganeh, P. Lopez-Crespo, Y.H. Tai, J.R. Yates, Locating the crack tip using displacement field data: A comparative study, Strain. 49 (2013) 102-115. https://doi.org/10.1111/str.12017.

[6] M. Mokhtarishirazabad, P. Lopez-Crespo, B. Moreno, A. Lopez-Moreno, M. Zanganeh, Evaluation of crack-tip fields from DIC data: A parametric study, Int. J. Fatigue. 89 (2016) 11-19. https://doi.org/10.1016/j.ijfatigue.2016.03.006.

[7] M.S. Kirugulige, H. V. Tippur, T.S. Denney, Measurement of transient deformations using digital image correlation method and high-speed photography: Application to dynamic fracture, Appl. Opt. 46 (2007) 5083-5096. https://doi.org/10.1364/AO.46.005083.

[8] S. Rabbolini, G.J. Pataky, H.Sehitoglu, S. Beretta, Anisotropic Stress Intensity Factor Ranges Measurements with DIC, Procedia Mater. Sci. 3 (2014) 2104-2110.

https://doi.org/10.1016/j.mspro.2014.06.340. 
[9] G.P. Mogadpalli, V. Parameswaran, Determination of stress intensity factor for cracks in orthotropic composite materials using digital image correlation, Strain. 44 (2008) 446-452.

https://doi.org/10.1111/j.1475-1305.2007.00391.x.

[10] D. Lee, H. Tippur, P. Bogert, Quasi-static and dynamic fracture of graphite/epoxy composites: An optical study of loading-rate effects, Compos. Part B Eng. 41 (2010) 462-474.

https://doi.org/10.1016/j.compositesb.2010.05.007.

[11] J.R.Rice, "A Path Independent Integral and the Approximate Analysis of Strain Concentration by Notches and Cracks," J. Appl. Mech. 35 (1968) 379.

[12] M.S. Dadkhah, A.S. Kobayashi, HRR field of a moving crack, an experimental analysis, Eng. Fract. Mech. 34 (1989) 253-262. https://doi.org/10.1016/0013-7944(89)90258-0.

[13] Y. Jiang, A. Akkus, R. Roperto, O. Akkus, B. Li, L. Lang, S. Teich, Measurement of J-integral in CAD/CAM dental ceramics and composite resin by digital image correlation, J. Mech. Behav. Biomed. Mater. 62 (2016) 240-246. https://doi.org/10.1016/j.jmbbm.2016.05.012.

[14] S. Yoneyama, S. Arikawa, S. Kusayanagi, K. Hazumi, Evaluating J-integral from displacement fields measured by digital image correlation, Strain. 50 (2014) 147-160.

https://doi.org/10.1111/str.12074.

[15] R.A. Cidade, D.S.V. Castro, E.M. Castrodeza, P. Kuhn, G. Catalanotti, J. Xavier, P.P. Camanho, Determination of mode I dynamic fracture toughness of IM7-8552 composites by digital image correlation and machine learning, Compos. Struct. 210 (2019) 707-714.

https://doi.org/10.1016/j.compstruct.2018.11.089.

[16] E. Breitbarth, T. Strohmann, M. Besel, S. Reh, Determination of stress intensity factors and J integral based on digital image correlation, Frat. Ed Integrita Strutt. 13 (2019) 12-25. https://doi.org/10.3221/IGF-ESIS.49.02.

[17] D.M. Montenegro, L.P. Canal, J. Botsis, M. Zogg, A.R. Studart, K. Wegener, On the validity of the Jintegral as a measure of the transverse intralaminar fracture energy of glass fiber-reinforced polyurethanes with nonlinear material behavior, Int. J. Solids Struct. 139-140 (2018) 15-28. https://doi.org/10.1016/j.ijsolstr.2018.01.019.

[18] G. Catalanotti, P.P. Camanho, J. Xavier, C.G. Dávila, A.T. Marques, Measurement of resistance curves in the longitudinal failure of composites using digital image correlation, Compos. Sci. Technol. 70 (2010) 1986-1993. https://doi.org/10.1016/j.compscitech.2010.07.022.

[19] S.G. Lekhnitskii, S.W. Tsai, T. Cheron, Anisotropic Plates, Third Edition, Gordon and Breach Science Publishers, 1987.

[20] S.T. Lin, Z. Feng, R.E. Rowlands, Thermoelastic Determination of Stress Intensity Factors in orthotropic composite using the J-Integral, Eng. Fract. Mech. 70 (2003) 367-369. https://doi.org/10.1016/s0013-7944(02)00124-8.

[21] G.C. Sih, P.C. Paris, G.R. Irwin, On cracks in rectilinearly anisotropic bodies, Int. J. Fract. Mech. 1 (1965) 189-203. https://doi.org/10.1007/BF00186854.

[22] G. Pitarresi, M. Ricotta, G. Menghetti, Investigation of the crack tip stress field in a stainless steel SENT specimen by means of Thermoelastic Stress Analysis, Procedia Struct. Integr. 00 (2019) 1-14. https://doi.org/10.1016/j.prostr.2019.08.173.

[23] K.H. Lee, J.S. Hawong, S.H. Choi, Dynamic stress intensity factors KI, KII and dynamic crack propagation characteristics of orthotropic material, Eng. Fract. Mech. 53 (1996) 119-140. https://doi.org/10.1016/0013-7944(95)00077-9. 
[24] C.F. Shih, B. Moran, T. Nakamura, Energy release rate along a three-dimensional crack front in a thermally stressed body, Int. J. Fract. 30 (1986) 79-102. https://doi.org/10.1007/BF00034019.

[25] T.L. Anderson, Fracture Mechanics, Fundamental and Applications, Fourth Edi, 2017.

[26] T.C. Suo, Z; Bao, G; Fan, B; Wang, Orthotropic Rescaling, Int. J. Solids Struct. 28 (1991) 235-248. https://doi.org/https://doi.org/10.1016/0020-7683(91)90208-W.

[27] B. Pan, K. Qian, H. Xie, A. Asundi, Two-dimensional digital image correlation for in-plane displacement and strain measurement: A review, Meas. Sci. Technol. 20 (2009). https://doi.org/10.1088/0957-0233/20/6/062001.

[28] B. Pan, Digital image correlation for surface deformation measurement: Historical developments, recent advances and future goals, Meas. Sci. Technol. 29 (2018). https://doi.org/10.1088/13616501/aac55b.

[29] V. Kibitkin, A. Solodushkin, V. Pleshanov, A. Napryushkin, On a choice of input parameters for calculation the vector field and deformation with DIC, Meas. J. Int. Meas. Confed. 95 (2017) 266272. https://doi.org/10.1016/j.measurement.2016.10.020.

[30] J.L. Pereira, J. Xavier, B. Ghiassi, J. Lousada, J. Morais, On the identification of earlywood and latewood radial elastic modulus of Pinus pinaster by digital image correlation: A parametric analysis, J. Strain Anal. Eng. Des. 53 (2018) 566-574. https://doi.org/10.1177/0309324718786351.

[31] P.P. Camanho, P. Maimí, C.G. Dávila, Prediction of size effects in notched laminates using continuum damage mechanics, Compos. Sci. Technol. 67 (2007) 2715-2727. https://doi.org/10.1016/j.compscitech.2007.02.005.

[32] MatchID ${ }^{\circledR}$ Metrology beyond colors. MatchID Documentation - http://matchid.eu/Documentation/, (2017).

[33] MATLAB 2019a, The MathWorks, Inc., Natick, Massachusetts, United States. Matlab Documentation - https://it.mathworks.com/help/matlab/, (2019).

[34] G.H. Erçin, P.P. Camanho, J. Xavier, G. Catalanotti, S. Mahdi, P. Linde, Size effects on the tensile and compressive failure of notched composite laminates, Compos. Struct. 96 (2013) 736-744. https://doi.org/10.1016/j.compstruct.2012.10.004.

[35] G. Catalanotti, A. Arteiro, M. Hayati, P.P. Camanho, Determination of the mode I crack resistance curve of polymer composites using the size-effect law, Eng. Fract. Mech. 118 (2014) 49-65. https://doi.org/10.1016/j.engfracmech.2013.10.021. 